Article

\title{
Design Optimization of a Dual-Bleeding Recirculation Channel to Enhance Operating Stability of a Transonic Axial Compressor
}

\author{
Tien-Dung Vuong (D) and Kwang-Yong Kim * (D)
}

check for

updates

Citation: Vuong, T.-D.; Kim, K.-Y Design Optimization of a Dual-Bleeding Recirculation Channel to Enhance Operating Stability of a Transonic Axial Compressor. Energies 2022, 15, 159. https://doi.org/ 10.3390/en15010159

Academic Editors: Davide Astolfi and Abdessattar Abdelkefi

Received: 4 November 2021 Accepted: 22 December 2021 Published: 27 December 2021

Publisher's Note: MDPI stays neutral with regard to jurisdictional claims in published maps and institutional affiliations.

Copyright: (C) 2021 by the authors. Licensee MDPI, Basel, Switzerland. This article is an open access article distributed under the terms and conditions of the Creative Commons Attribution (CC BY) license (https:// creativecommons.org/licenses/by/ $4.0 /)$
Department of Mechanical Engineering, Inha University, Incheon 22212, Korea; vuongtien97bkhn@gmail.com * Correspondence: kykim@inha.ac.kr; Tel.: +82-32-872-3096

\begin{abstract}
The present work performed a comprehensive investigation to find the effects of a dualbleeding port recirculation channel on the aerodynamic performance of a single-stage transonic axial compressor, NASA Stage 37, and optimized the channel's configuration to enhance the operating stability of the compressor. The compressor's performance was examined using three parameters: The stall margin, adiabatic efficiency, and pressure ratio. Steady-state three-dimensional Reynoldsaveraged Navier-Stokes analyses were performed to find the flow field and aerodynamic performance. The results showed that the addition of a bleeding channel increased the recirculation channel's stabilizing effect compared to the single-bleeding channel. Three design variables were selected for optimization through a parametric study, which was carried out to examine the influences of six geometric parameters on the channel's effectiveness. Surrogate-based design optimization was performed using the particle swarm optimization algorithm coupled with a surrogate model based on the radial basis neural network. The optimal design was found to increase the stall margin by $51.36 \%$ compared to the case without the recirculation channel with only $0.55 \%$ and $0.28 \%$ reductions in the peak adiabatic efficiency and maximum pressure ratio, respectively.
\end{abstract}

Keywords: axial compressor; recirculation channel; RANS analysis; optimization; stall margin; genetic algorithm

\section{Introduction}

An axial compressor is an integral component of gas turbines, which powers the majority of today's aircrafts. When compressors operate at the maximum pressure ratio, they are prone to a phenomenon called a stall. A stall is a detrimental occurrence that destabilizes the flow field and greatly degrades performance and safety. In axial compressors, a primary cause of stall is the tip leakage vortex occurring in the rotors' tip region. At a high pressure ratio, the tip leakage vortex breaks down due to its interaction with the passage shock wave. As a result, a stagnation zone containing low-momentum airflow is formed, which acts as a blockage in the flow field. As the pressure continues to increase, the blockage grows until it destabilizes the whole flow path, and the compressor falls into a stall. With a rising demand for higher-pressure-ratio axial compressors, the problem associated with the tip leakage vortex becomes more concerning and thus, increasing efforts are being spent to solve this issue.

A promising solution to improve the stability of axial compressors is the use of a recirculation channel. A design schematic of a basic recirculation channel can be found in the work of Hathaway [1]. When using a recirculation channel, air flow is bled from a downstream location on the compressor shroud and then re-injected to an upstream location. Due to the pressure difference between the bleeding and injecting positions, the accelerated recirculating air re-energizes the low-momentum air inside the blockage. Consequently, the expansion of the blockage is delayed, and stall inception is prevented.

Several designs of recirculation channels have been proposed and patented so far [2-4]. A basic concept of self-recirculating casing treatment was presented by Hathaway [1], who 
combined air bleeding and injection in the rotors' tip region. The stable range extension of the tested transonic fan, NASA Rotor 67 , was increased by $225 \%$ with inlet distortion while injection location and angle were found to be critical design parameters. A stagerecirculation design from Strazisar et al. [5] extracted air from a stator casing and reinjected it to the upstream of a preceding rotor, which extended the stable operating range of the transonic compressor NASA Stage 35. They concluded that increasing the circumferential mass-averaged axial velocity at the rotor tip is the key to improving the compressor's stability. An end-wall recirculation scheme with an anti-swirl vaned passage was tested by Khaleghi et al. [6] on the high-speed fan, NASA Rotor 67. The scheme was able to increase the stable range extension with a very small penalty in efficiency. In a design of flow recirculation, Weichert et al. [7] adapted the recirculating flow rate to the compressor's working condition. The recirculating air flow was minimized at the compressor's design condition while maximized at the near-stall condition, which led to a stall margin improvement in the range of 2.2-6.0\%. A recirculation scheme of Khaleghi [8] enhanced the performance of the high-speed fan, NASA Rotor 67, by using 22 skewed channels. The increased stability was found to be the result of recirculating flow pushing the tip leakage vortex and passage shock downstream at the near-stall condition.

The self-recirculation concepts suggested by Hathaway [1] and Strazisar [5] were parameterized and tested by Wang et al. [9] focusing on the circumferential coverage and injector's throat height. The results indicated that optimizing recirculation channel designs could improve not only compressors' stability, but also other performance parameters. A feedback channel design proposed by Dinh et al. [10] enhanced the stall margin of the singlestage transonic compressor, NASA Stage 37 by $26.8 \%$ with only a $0.14 \%$ decrease in the efficiency. Unlike other recirculation schemes, their channel was located between the rotors' edges, which facilitated its integration into multistage configurations. The effectiveness of flow recirculation channels on both single- and multi-stage compressors was experimentally investigated by Li et al. [11]. Application of the recirculation channels increased the stall margins of the single- and three-stage compressors by $13.7 \%$ and $13.0 \%$, respectively, without a reduction in the efficiency. A recirculation casing treatment introduced by Khaleghi [12] extracted air from downstream and circumferentially re-injected it into the flow path in the opposite direction of the blade rotation. The results showed a considerable extension in the compressor's stable working range at the expense of a moderate efficiency loss. Recently, a casing treatment combining flow recirculation and circumferential groove was proposed by Vuong et al. [13] to enhance the stability of the transonic compressor, NASA Stage 37. The design was able to provide a maximum stall margin increase of $42.5 \%$ with a less than $1 \%$ decrease in the adiabatic efficiency.

In the last couple of decades, surrogate-based optimization techniques have shown great potential in the design of turbomachinery. Considering the complexity of turbomachine design, optimization techniques have contributed greatly to speeding up the design process and discovering unexpected designs. The blade shape of a transonic axial compressor was optimized by Samad and Kim [14] using the elitist non-dominated sorting genetic algorithm (NSGA-II) [15] combined with response surface approximation [16]. With the two extreme-end designs of the Pareto front, two objective functions, i.e., the total pressure ratio and adiabatic efficiency, were increased by $1.76 \%$ and $0.41 \%$, respectively. Using the hybrid multi-objective evolutionary algorithm (MOEA) [17], Kim et al. [18] attempted to enhance the efficiency and mitigate the noise level of an axial fan. The NSGA-II algorithm and RBNN model were utilized by Khalfallah et al. [19] to find an optimal design of a centrifugal compressor to maximize the efficiency and stall margin. Various optimization algorithms coupled with the RBNN model were implemented by Ma et al. [20] to find an optimal ring cavity design for stall margin improvement of a centrifugal compressor. Among four popular algorithms, i.e., the genetic algorithm (GA) [21], particle swarm optimization (PSO) [22], simulated annealing [23], and SQP, PSO was found to be the most effective one in terms of the computing time and stall margin gain. Cheng et al. [24] successfully applied an improved artificial bee colony algorithm [25] to find an optimal blade shape 
for a four-stage, low-pressure axial compressor. The optimized blade raised the adiabatic efficiency at the design point from $82.07 \%$ to $82.55 \%$ and stall margin from $10.2 \%$ to $15.0 \%$.

In the present work, the performance of a recirculation channel design with two bleeding channels (RE2B) was analyzed and optimized to enhance the stability of the singlestage transonic axial compressor, NASA Stage 37. In comparison with the conventional single-bleeding design, the addition of one more bleeding channel was expected to increase the amount of recirculating airflow as well as the energy it contains, which would improve the stabilizing function of the channel. A previous study on a similar recirculation casing treatment design [26] has also confirmed its positive influence on a transonic compressor's stability. However, no comprehensive investigation of the casing treatment design has been presented in the literature. Therefore, in this study, a detailed parametric study with six geometric parameters of the channel was conducted to assess their levels of influence on the channels' effectiveness. Moreover, based on this parametric study, a surrogate-based design optimization was performed to find an optimal channel design with maximized stability improvement. Three-dimensional Reynolds-averaged Navier-Stokes (RANS) equations were solved numerically for the aerodynamic analysis.

\section{Compressor Model and Casing Treatment Design}

The single-stage transonic axial compressor tested in the present work was NASA Stage 37. The report of Reid and Moore [27] provided detailed information regarding the compressor's geometry and aerodynamic performance. This compressor exhibits a spike-type rotating stall that originated from the rotors' tip [28,29], which makes it wellsuited for the investigation of casing treatments. The compressor comprises 36 rotor blades rotating at a design speed of $17,185.7 \mathrm{rpm}$ and 46 stationary stator blades. The tip clearance values are $0.0400 \mathrm{~cm}$ under the rotor shroud and $0.0762 \mathrm{~cm}$ over the stator hub, which correspond to $1.44 \%$ of the axial chord length at the rotor tip and $2.11 \%$ of the axial chord length at the stator tip, respectively. From the experiment by Reid and Moore [27], when running at $100 \%$ design speed and the peak efficiency condition, the compressor's total pressure ratio and adiabatic efficiency were $2.00 \%$ and $84.00 \%$, respectively, with a mass flow rate of $20.74 \mathrm{~kg} / \mathrm{s}$. At the near-stall condition, the compressor's total pressure ratio was 2.093 and the mass flow rate was $19.60 \mathrm{~kg} / \mathrm{s}$. The mass flow rate reached $20.93 \mathrm{~kg} / \mathrm{s}$ at the choking condition. The reference pressure and temperature were $101,325 \mathrm{~Pa}$ and $288.15 \mathrm{~K}$, respectively.

The schematic of RE2B is illustrated in Figure 1. The recirculation channels are located on the shroud between the leading and trailing edges of the rotor blades. Compared to the previously mentioned similar design [26] that positioned its injection and bleeding ports outside the rotor's edges, the present design does not intrude into other blade row regions and is more lightweight. The whole system consists of 36 channels uniformly distributed around the compressor's annulus, each of which covers $8^{\circ}$ in the circumferential direction. The spacing between individual channels $\left(2^{\circ}\right)$ is necessary in practice for the electrical wiring around the compressor's casing. The design of the RE2B channel is parameterized using six geometric parameters: The injection location $\left(\mathrm{L}_{\mathrm{F}}\right)$, injection angle $(\alpha)$, middle bleed location $\left(\mathrm{L}_{\mathrm{M}}\right)$, middle bleed angle $(\beta)$, rear bleed location $\left(\mathrm{L}_{\mathrm{B}}\right)$, and rear bleed angle $(\gamma)$. Their values for the reference design are listed in Table 1 , with all the location parameters non-dimensionalized by the rotor tip's axial chord length $\left(C_{R}\right)$. 


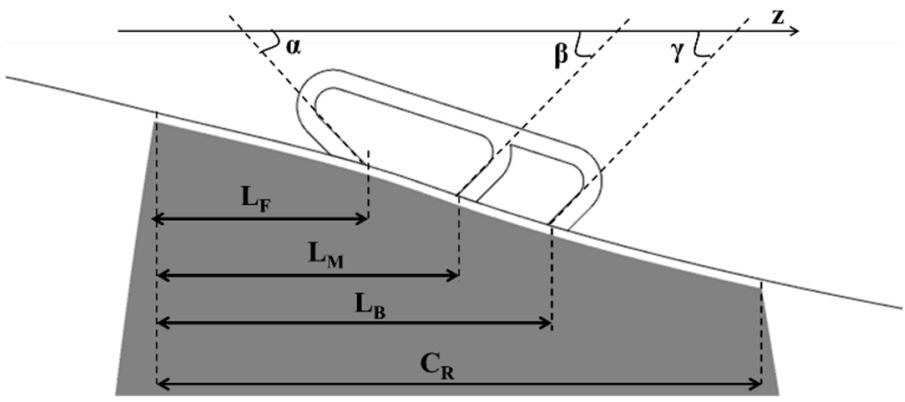

(a)

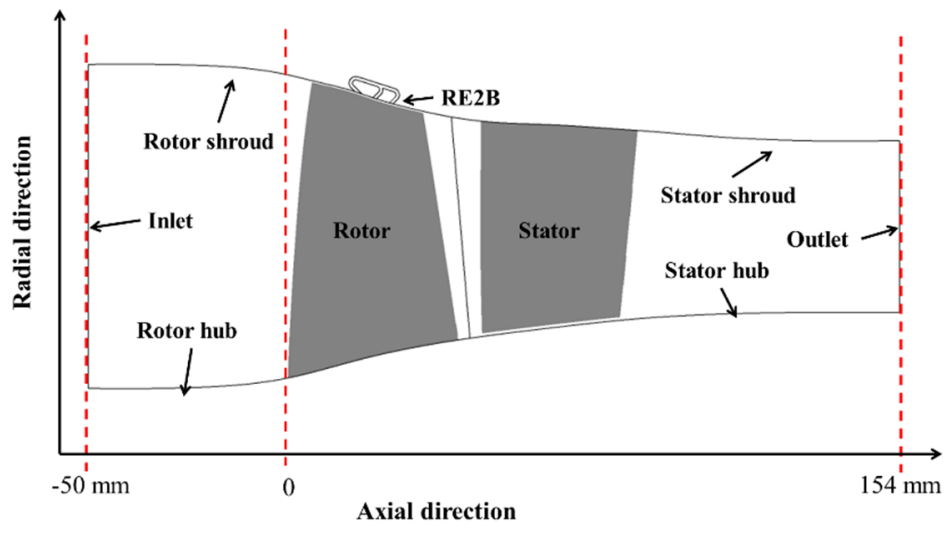

(b)

Figure 1. Geometric description of NASA Stage 37 compressor and RE2B: (a) Geometric parameters of RE2B; (b) meridional view of NASA Stage 37 with RE2B.

Table 1. Reference design of RE2B.

\begin{tabular}{ccccccc}
\hline Parameters & $\mathrm{L}_{\mathrm{F}} / \mathrm{C}_{\mathrm{R}}(\mathbf{\%})$ & $\mathrm{L}_{\mathrm{M}} / \mathrm{C}_{\mathbf{R}}(\mathbf{\%})$ & $\mathrm{L}_{\mathrm{B}} / \mathrm{C}_{\mathbf{R}}(\mathbf{\%})$ & $\boldsymbol{\alpha}\left({ }^{\circ}\right)$ & $\boldsymbol{\beta}\left({ }^{\circ}\right)$ & $\gamma\left({ }^{\circ}\right)$ \\
\hline Values & 45 & 55 & 70 & 45 & 45 & 45 \\
\hline
\end{tabular}

\section{Numerical Analysis}

\subsection{Numerical Methods}

The aerodynamic analysis was conducted using the commercial code ANSYS CFX $15.0^{\circledR}$ [30]. To reduce the computing time, only one compressor's passage comprising one rotor and stator pair was considered in the computation. As illustrated in Figure 2, the computational domain comprises three sub-domains: The rotor, stator, and RE2B channel. A hexahedral mesh system for the computational domain was made using TurboGrid ${ }^{\circledR}$ and ICEM-CFD ${ }^{\circledR}$. TurboGrid ${ }^{\circledR}$ was used to generate the mesh for the rotor and stator blocks (more information can be found in ref. [10]). The mesh of RE2B was created using $\mathrm{ICEM}_{-\mathrm{CFD}}{ }^{\circledR}$ with the determinant quality kept larger than 0.7. Assigning the boundary conditions, solving the governing equations, and post-processing the numerical results were performed by ANSYS CFX-Pre, CFX-Solver, and CFD-Post, respectively.

All simulations were performed using steady RANS analysis in the present work. For the steady analysis, the rotating and stationary blocks were linked by a general grid interface (GGI) method. The frozen-rotor method was applied at the interface between the rotor and stator domains $\left(360^{\circ} / 36=10^{\circ}\right.$ for the rotor, $360^{\circ} / 46=7.826^{\circ}$ for the stator) and also between RE2B and rotor domains ( $8^{\circ}$ for the channel and $10^{\circ}$ for the rotor). The influences of the frozen-rotor method on the numerical results in comparison with those of the stage method were discussed by Shim and Kim [31]. 


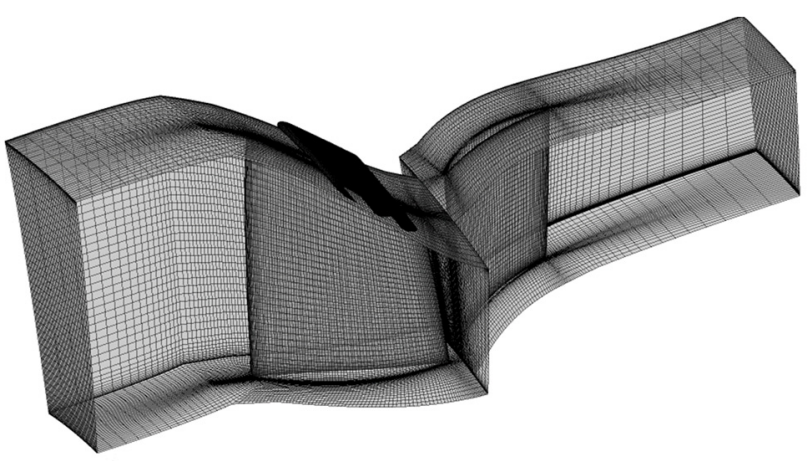

Figure 2. Computational domain and grid system.

The $k-\varepsilon$ turbulence model was used with a scalable wall function, while $y+$ values of the first nodes near the walls were maintained in a range of 20-100. A fully implicit element-based finite volume method was implemented to discretize three-dimensional (3D) governing differential equations. For the advection terms, a high-resolution scheme using the principles of Barth and Jesperson [32] was used, which has second-order accuracy in space.

The simulations used ideal gas (air) as the working fluid. At the stator's outlet, the average static pressure condition was used, while at the rotor's inlet, the turbulence intensity and total temperature were specified as $5 \%$ and $288.15 \mathrm{~K}$, respectively. Smooth and adiabatic conditions were applied to all wall boundaries. The side boundaries of the rotor and stator domains were specified with periodic conditions.

In this work, the numerical calculations were performed until the near-stall point, which shows the highest pressure ratio. Due to the intrinsic unsteady characteristics of the stall/surge phenomenon, strict criteria for the near-stall point must be implemented to achieve a reasonably accurate assessment with steady simulation. The convergence criteria used in this work were those suggested by Chen et al. [33]: The variation between the inlet and outlet mass flow rates is less than $0.3 \%$, the inlet mass flow rate fluctuation is less than $0.001 \mathrm{~kg} / \mathrm{s}$ for 300 steps, and the change of adiabatic efficiency is less than $0.3 \%$ per 100 steps.

To obtain the performance curve of the compressor, at the compressor's outlet, the average static pressure was varied from the choking condition $(0 \mathrm{~Pa})$ to the near-stall condition with a step size of $100 \mathrm{~Pa}$ around the peak efficiency condition and a step size of $10 \mathrm{~Pa}$ (which corresponds to about $0.0001 \mathrm{~kg} / \mathrm{s}$ of mass flow rate) at the near-stall condition. The convergence criterion for the root-mean-square residual of each governing equation was limited to $10^{-6}$. The calculations were carried out by a computer with an Intel i7-4930K 3.4 GHz CPU. Each operating point was obtained after 2-3 h simulation time, and it took about $70 \mathrm{~h}$ to find a performance curve on average.

\subsection{Performance Parameters}

Three parameters, i.e., the total pressure ratio $(P R)$, adiabatic efficiency $(\eta)$, and stall margin $(S M)$, were used to assess the aerodynamic performance and operational stability of the transonic compressor with and without the casing treatment $[10,34,35]$ :

$$
\begin{gathered}
\text { PR }=\frac{p_{t, \text { out }}}{p_{t, \text { in }}} \\
\eta=\frac{\left(\frac{p_{t, \text { out }}}{p_{t, \text { in }}}\right)^{\frac{\gamma-1}{\gamma}}-1}{\frac{T_{t, \text { out }}}{T_{t, \text { in }}}-1} \times 100 \% \\
S M=\left(\frac{P R_{N S}}{P R_{\text {peak }}} \times \frac{\dot{m}_{\text {peak }}}{\dot{m}_{N S}}-1\right) \times 100 \%
\end{gathered}
$$


where $p_{t}, T_{t}$, and $\dot{m}$ are the total pressure, total temperature, and normalized mass flow rate, respectively. The subscripts in and out indicate the values measured at the compressor's inlet and outlet, respectively. The subscripts peak and NS refer to the peak adiabatic efficiency and near-stall condition, respectively. The subscripts $S C$ and $C T$ refer to the smooth casing and casing treatment, respectively.

\subsection{Validation and Grid-Dependency Test}

The validation of the numerical results for the NASA Stage 37 compressor was presented in detail in the study of Dinh et al. [10]. Their work showed that the numerical results closely reproduced the performance characteristic compared to the experimental data [28] as shown in Figure 3. Since the same compressor model and numerical methods were used in this work, this validates the accuracy of the subsequent numerical results.

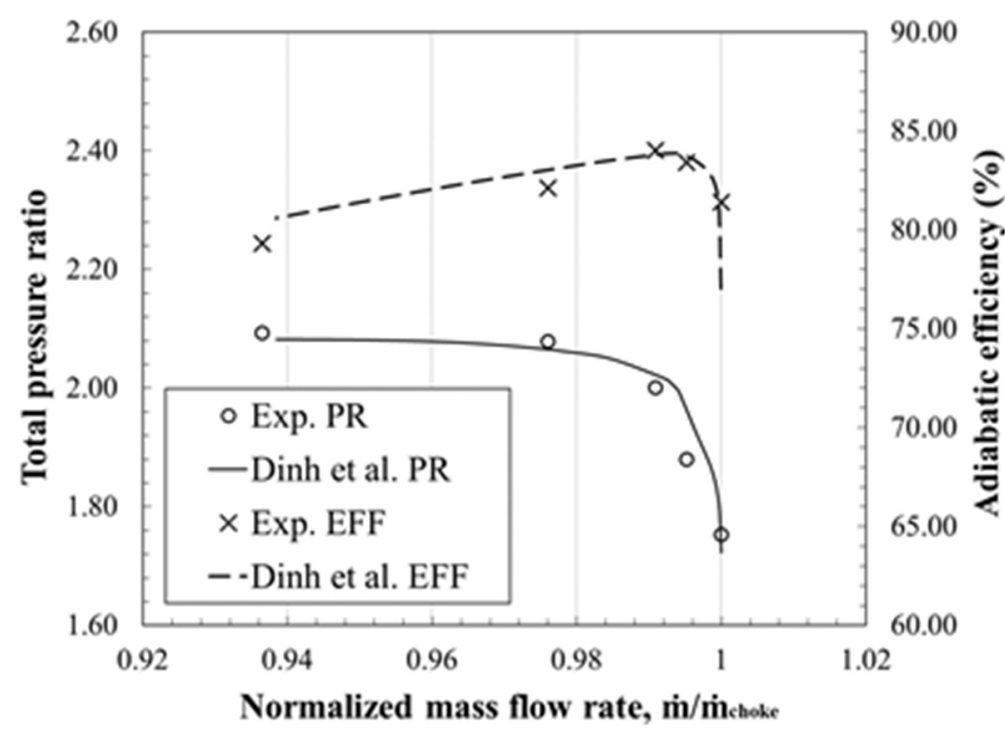

Figure 3. Validation of numerical results for NASA Stage 37 compressor model [10] against experimental measurement [27].

To evaluate the grid-dependency of the numerical results with RE2B, analysis using the grid convergence index (CGI) based on the Richardson extrapolation technique was performed. This analysis was proposed by Celik and Karatekin [36] and is widely used to assess the grid dependency of numerical solutions.

Details of the CGI analysis of RE2B's meshes are presented in Table 2. As shown in this table, the stall margin value converges monotonically as the number of mesh elements increases. From these results, mesh G1 was selected for all proceeding calculations. The grid system in the compressor without RE2B is the same as that used by Dinh et al. [10] (340,556 nodes for rotor block and 249,524 nodes for stator block).

\subsection{Optimization Methods}

The single-objective optimization problem is defined as follows:

$$
\begin{gathered}
\text { Maximize } \boldsymbol{F}(\boldsymbol{x}) \\
x_{i}^{L} \leq x_{i} \leq x_{i}^{U}
\end{gathered}
$$

where $\boldsymbol{F}$ is an objective function and $x\left(=\left\{x_{i}\right\}\right)$ is a vector of $\mathrm{n}$ design variables. An optimization algorithm performs a search procedure to find an optimal solution within the design space specified by the lower limit $x_{i}^{L}$ and upper limit $x_{i}^{U}$ of each design variable. The stall margin was selected as the objective function for optimization in the present study:

$$
F=S M
$$


Table 2. CGI analysis results for the grid in RE2B.

\begin{tabular}{ccc}
\hline Parameters & Symbols & Values \\
\hline $\begin{array}{c}\text { Number of mesh elements } \\
\text { in RE2B }\end{array}$ & G1/G2/G3 & $293,319 / 148,665 / 63,632$ \\
\hline Grid refinement factors & $\mathrm{r} 21 / \mathrm{r} 32$ & $1.97 / 2.34$ \\
\hline $\begin{array}{c}\text { Stall margin corresponding to } \\
\text { G1/G2/G3 }\end{array}$ & $\mathrm{SM} 1 / \mathrm{SM} 2 / \mathrm{SM} 3$ & $13.40 / 13.38 / 13.24$ \\
\hline Apparent order & $\mathrm{p}$ & 3.13 \\
\hline Extrapolated value & $\phi_{\text {ext }}^{21}$ & 13.40 \\
\hline Approximated relative error & $e_{a}^{21}$ & $0.0746 \%$ \\
\hline Extrapolated relative error & $e_{\text {ext }}^{21}$ & $0.0101 \%$ \\
\hline Grid convergence index & $C G I_{\text {fine }}^{21}$ & $0.0126 \%$ \\
\hline
\end{tabular}

The design of experiment (DOE) was performed using the Latin hypercube sampling (LHS) method [37]. Sampling points (or design points) are generated by LHS and are composed of an $i \times j$ matrix, where $i$ and $j$ represent the numbers of sampling points and design variables, respectively. Each column $j$ is filled with a randomly paired permutation level of $1,2, \ldots, i$ to form a Latin hypercube. The method is provided as the function lhsdesign in MATLAB [38].

The objective function was approximated by surrogate modeling using RBNN. RBNN is a two-layer neural network that comprises a hidden layer of the radial basis function and a linear output layer. The two hyper parameters of this surrogate model are an error goal and a spread constant. The former's value can be chosen by the user based on the permissible error from the mean input response. MATLAB provides a built-in function, newrb, to apply RBNN modeling [38].

PSO [25] is a meta-heuristic optimization algorithm that replicates the "swarm behavior" of a bird flock or fish school. The algorithm starts with a population, called a swarm, of particles, each of which is a possible solution. These particles are then moved around the search space following some defined formulae. The movement of each particle is under the influence of its own best-known position (local best objective function value) and the whole swarm's best-known position (global best objective function value). Following this principle, the particles are guided toward a better position after each iteration. The process is repeated until a solution that satisfies all conditions is found. In MATLAB, the single-objective PSO is provided in the optimization toolbox and can be utilized by using function pso [38]. In the current study, the swarm size is set at 100 particles and the objective function tolerance is set at $10^{-6}$. The optimization procedure can be summarized as in Figure 4. 


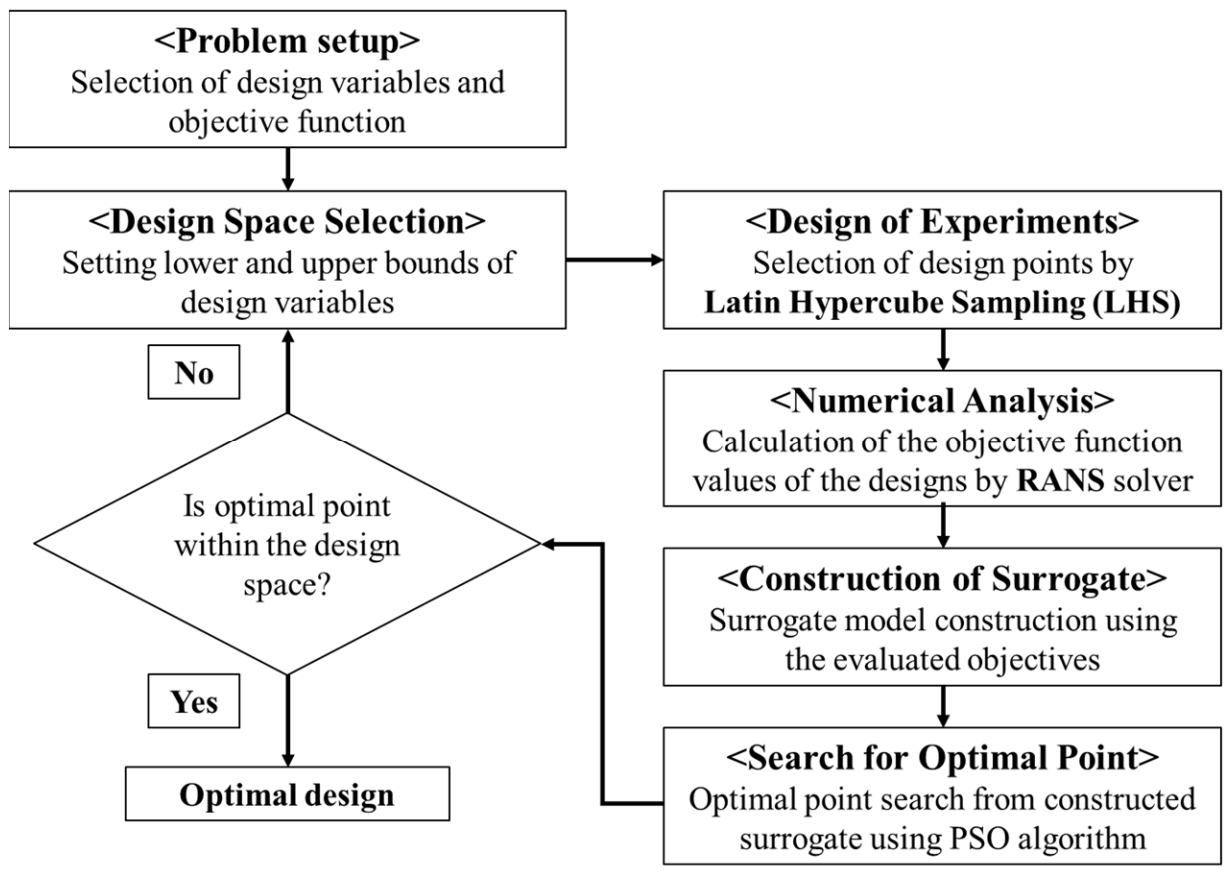

Figure 4. Single-objective optimization process.

\section{Results and Discussion}

\subsection{Results with the Reference Design of RE2B}

Figure 5 compares three performance curves of the compressor for the cases without any casing treatment (SC), with the conventional recirculation channel (RE) and with the reference RE2B channel (Table 1). As shown in the figure, the working mass flow range of the compressor is extended to lower mass flow rates when using both casing treatments. However, RE2B lowers the near-stall mass flow rate to the normalized value of 0.9179 , which is lower than that of RE (0.9198). In exchange, the losses in efficiency and the pressure ratio are higher with $\mathrm{RE} 2 \mathrm{~B}$ compared to those of $\mathrm{RE}$ at high mass flow rates, but these differences diminish for lower mass flow rates. Consequently, the difference between the pressure ratios at peak adiabatic efficiency and the near-stall condition increases with the RE2B design. In Equation (3), the stall margin value is affected by this difference in the pressure ratio and the corresponding mass flow range. Therefore, RE2B increases the stall margin from $9.95 \%$ of SC to $13.40 \%$, while RE only yields a stall margin of $12.37 \%$. This stall margin increase with RE2B is significant (about 34.7\% compared to that of smooth casing), at the expense of only $0.52 \%$ and $0.99 \%$ reductions in adiabatic efficiency and the pressure ratio at the peak efficiency condition, respectively. The following discussion explains the effects of RE2B on the compressor's performance by analyzing the flow field.
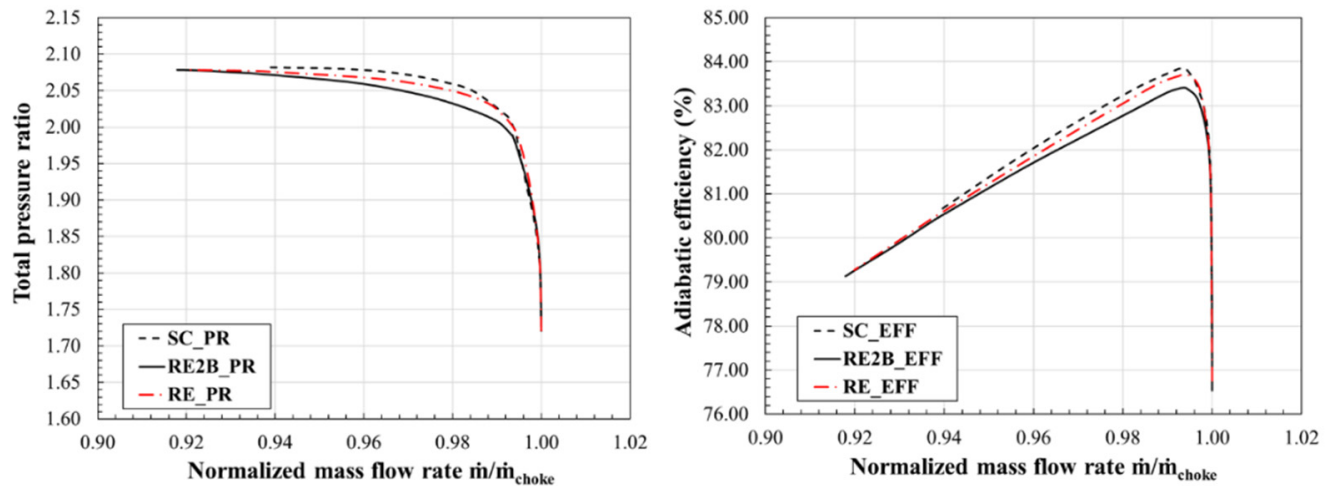

Figure 5. Performance curves for three configurations. 
Figure 6 illustrates streamlines of the tip leakage vortex at the near-stall condition. When either RE or RE2B is used, it can be seen that the tip leakage vortex is cut off from a portion of the leakage flow at the injection position. According to the numerical results, the leakage mass flow rate through the rotor's tip region is reduced from $0.00569 \mathrm{~kg} / \mathrm{s}$ of SC to $0.00539 \mathrm{~kg} / \mathrm{s}$ and $0.00534 \mathrm{~kg} / \mathrm{s}$ of RE and RE2B, respectively. This reduction of leakage flow indicates that the expansion of the blockage formed by the vortex breakdown is delayed, which contributes to the delay in the stall inception. There are three mechanisms related to RE and RE2B: (1) The injected flow pushes the leakage flow at the injection location to the main flow direction, (2) the leakage flow is ingested into the channel by the bleeding ports, and (3) a flow barrier is created in the tip gap by the recirculating flow from the injection to bleeding positions. As a result, the flow supply to the tip leakage vortex reduces. The decrease in the leakage flow with RE2B is higher than that with RE due to the additional bleeding channel, which increases the amount of bled and re-injected air flow. It is noted that a new vortex is formed in the case of RE2B. However, since it is located behind the passage shock, it does not suffer vortex breakdown and thus does not have a substantial destabilizing effect to induce stall inception.
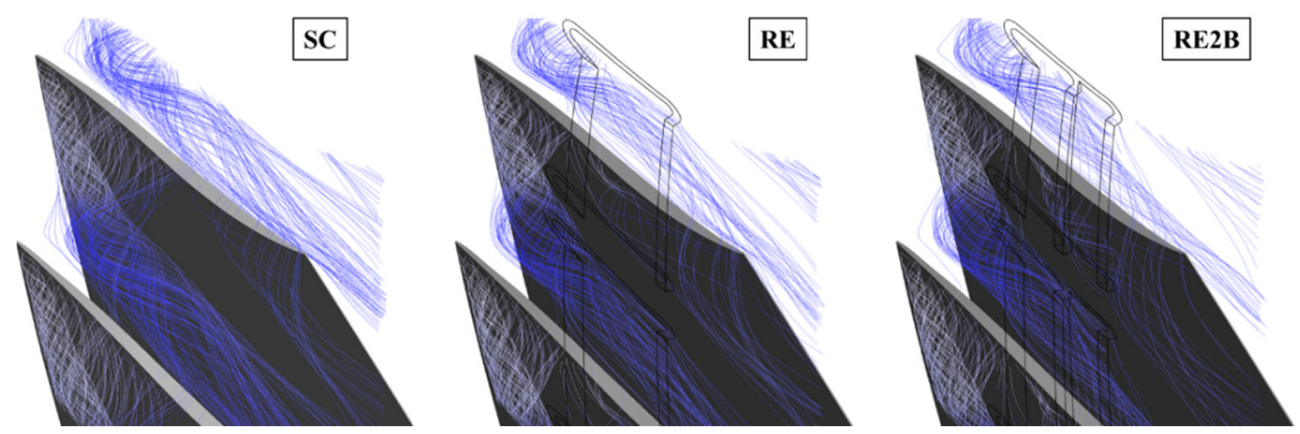

Figure 6. Streamlines of tip leakage vortex at near-stall condition.

Figure 7 shows streamlines near the suction surface of the rotor blade at the near-stall condition. The red dashed line indicates the lines of flow separation on the suction surface, which is pushed downward due to the recirculation channels. Consequently, the interaction between the tip leakage vortex and flow separation is reduced, which provides a more stable flow field in the tip region.
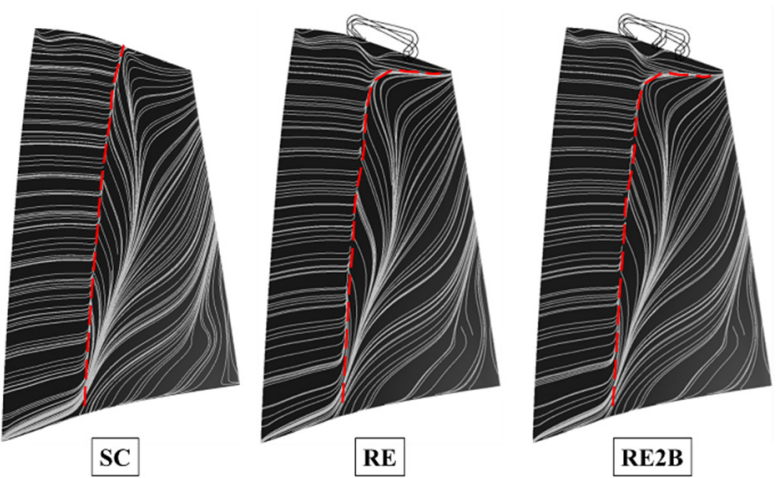

Figure 7. Streamlines on suction surface of rotor blade at near-stall condition.

In Figure 8, another advantage of RE2B over RE is observed. When using RE, a region of low speed flow, which might be a result of flow separation, is found over the rear-half of the rotor's suction surface. However, this stagnation zone disappears in the case with $\mathrm{RE} 2 \mathrm{~B}$, which reduces the blockage in the flow passage. While the stagnation zones in the stator region are larger with $\mathrm{RE} 2 \mathrm{~B}$, their negative effects on the stability are expected to be outweighed by the positive changes in the rotor region. 


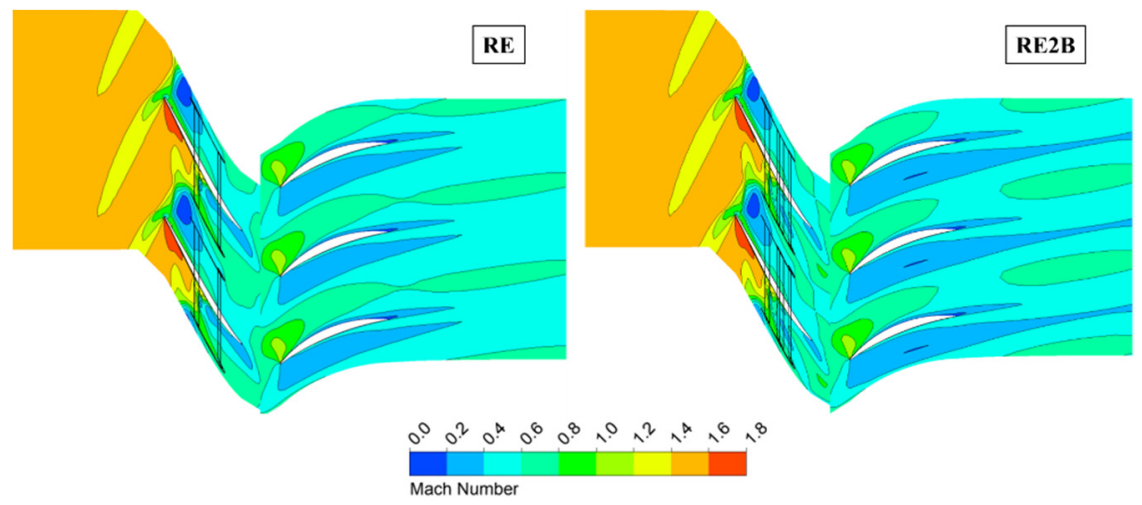

Figure 8. Relative Mach number contours at $98 \%$ rotor blade span at near-stall condition.

Figure 9 shows contours of circumferential-averaged static entropy at the peak adiabatic efficiency condition. The reason for the increased loss in efficiency is found to be the increase in entropy generation. In Figure 9, a region of high entropy emerges at the rotor tip region downstream of the recirculation channel. This is probably due to the formation of the secondary leakage vortex, which unavoidably induces efficiency loss.
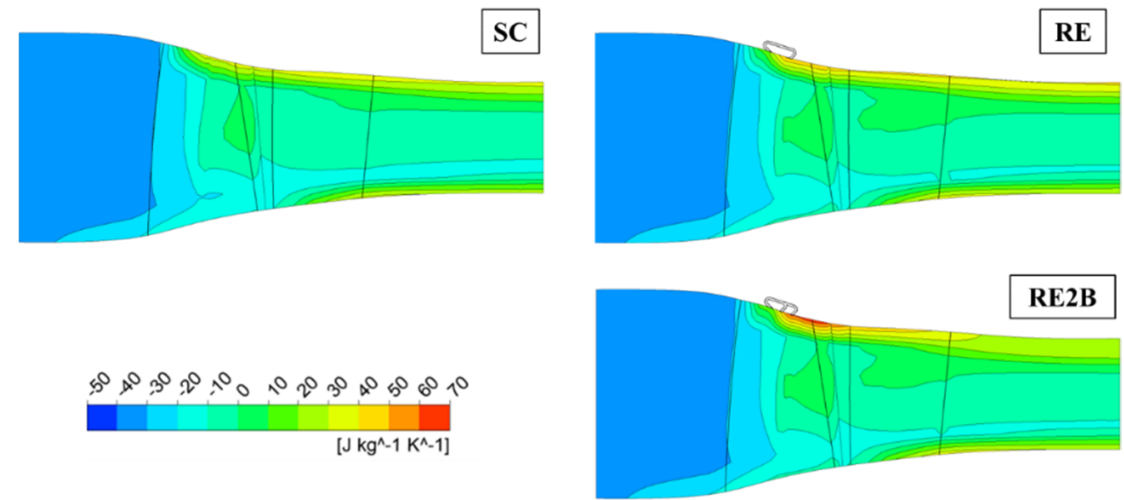

Figure 9. Circumferential-averaged static entropy contours at peak adiabatic efficiency condition.

\subsection{Parametric Study}

To determine the most influential variables in the channel's design, a parametric study was performed with six geometric parameters of RE2B. Their tested ranges are specified in Table 3. The reference values in Table 1 are maintained for the parameters that are not changed.

Table 3. Tested values of six geometric parameters.

\begin{tabular}{ccccccc}
\hline Parameters & $\mathbf{L}_{\mathbf{F}} / \mathbf{C}_{\mathbf{R}}(\mathbf{\%})$ & $\mathbf{L}_{\mathbf{M}} / \mathbf{C}_{\mathbf{R}}(\mathbf{\%})$ & $\mathbf{L}_{\mathbf{B}} / \mathbf{C}_{\mathbf{R}}(\mathbf{\%})$ & $\boldsymbol{\alpha}\left(^{\circ}\right)$ & $\beta\left(^{\circ}\right)$ & $\gamma\left({ }^{\circ}\right)$ \\
\hline Lower limit & 40 & 50 & 65 & 30 & 15 & 15 \\
Upper limit & 50 & 60 & 80 & 75 & 60 & 60 \\
\hline
\end{tabular}

Figure 10 presents the effects of injection location on the performance of the compressor. The highest stall margin of $13.40 \%$ is achieved at $\mathrm{L}_{\mathrm{F}} / \mathrm{CR}=45 \%$ while the effect on the peak adiabatic efficiency is relatively small. The influence of these parameters on the pressure ratio shows different trends for near-stall and peak efficiency conditions. In Figure 11, the effects of injection angle on the performance are illustrated. The stall margin reaches its peak at $\alpha=60^{\circ}$ while the best angle for efficiency is $45^{\circ}$. Increasing the angle from $45^{\circ}$ slightly reduces the pressure ratio at peak efficiency conditions but increases the pressure ratio at the near-stall condition. 

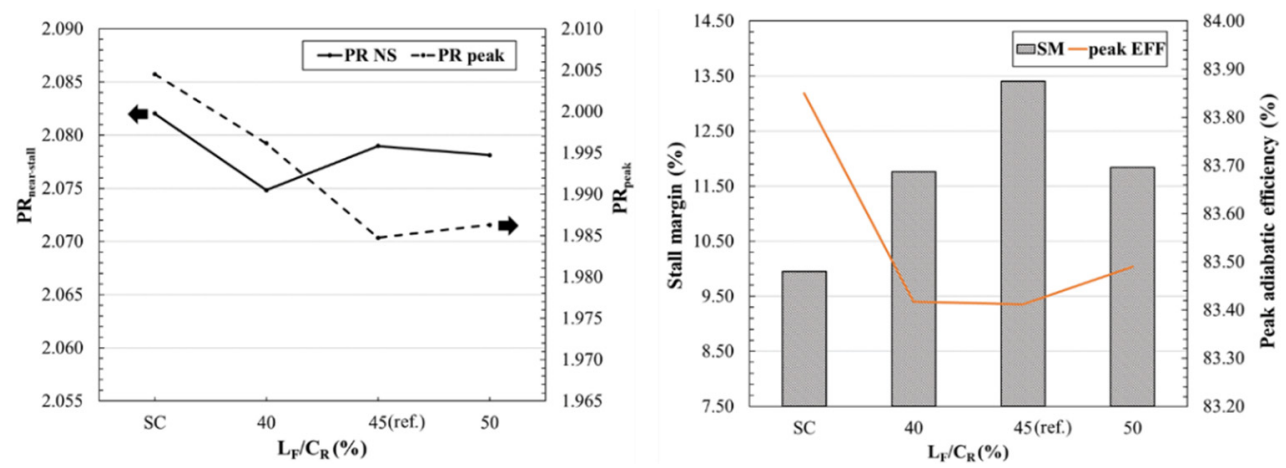

Figure 10. Effects of injection location on performance.
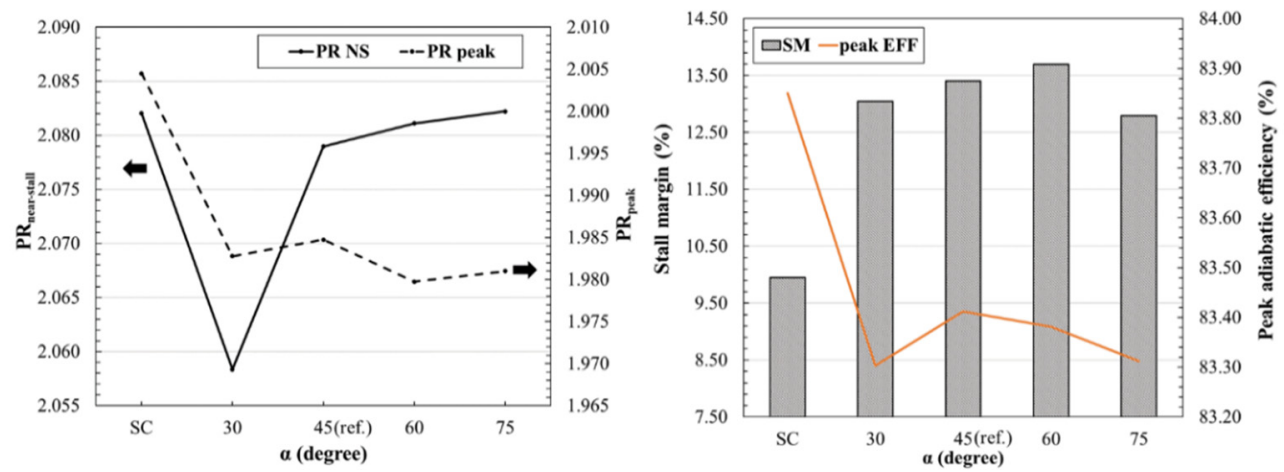

Figure 11. Effects of injection angle on performance.

Figure 12 shows that the best middle bleed location for stall margin improvement is $\mathrm{L}_{\mathrm{M}} / \mathrm{C}_{\mathrm{R}}=55 \%$. Moving the middle bleed port downstream slightly reduces the peak adiabatic efficiency. For the pressure ratio, a similar trend to that of the injection location is observed. Figure 13 presents the effects of the middle bleed angle on the performance. Overall, this angle has a small impact on all performance parameters. In terms of the stall margin, $\gamma=15^{\circ}$ provides the best value of $13.66 \%$.
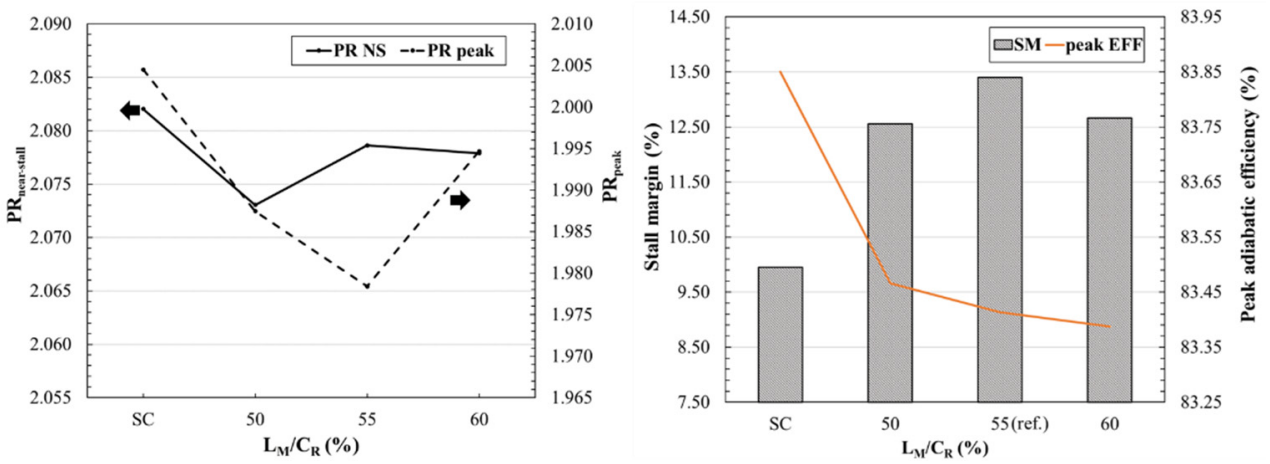

Figure 12. Effects of middle bleed location on performance. 

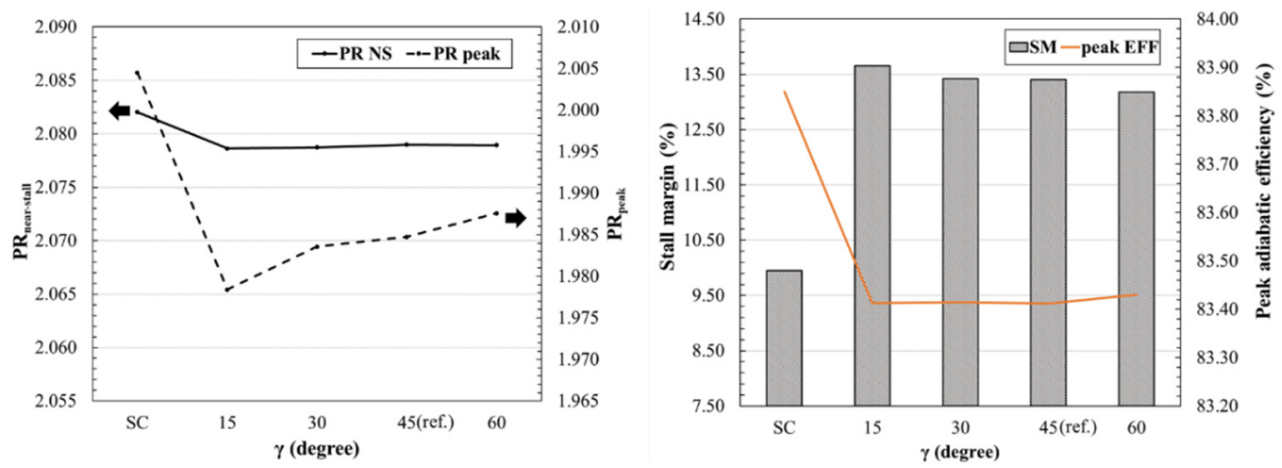

Figure 13. Effects of middle bleed angle on performance.

The effects of the rear bleed location on the performance are shown in Figure 14. $\mathrm{L}_{\mathrm{B}} / \mathrm{C}_{\mathrm{R}}=75 \%$ provides the best stall margin of $14.69 \%$ and the best peak adiabatic efficiency of $83.45 \%$. While the pressure ratio at peak efficiency conditions fluctuates, shifting the rear bleed location downstream reduces the pressure ratio in the examined range. Figure 15 presents the effects of the rear bleed angle on the performance. At $\beta=45^{\circ}$, the stall margin reaches the highest value of $13.40 \%$. Increasing the angle mitigates the loss in peak adiabatic efficiency, and increases the pressure rise at the peak efficiency condition while reducing the pressure rise at the near-stall point.
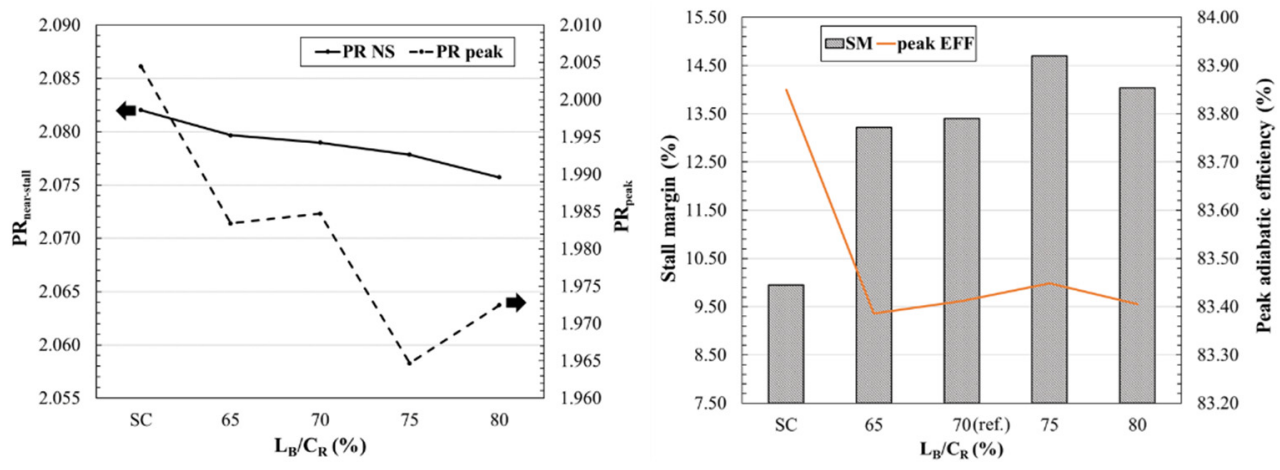

Figure 14. Effects of rear bleed location on performance.
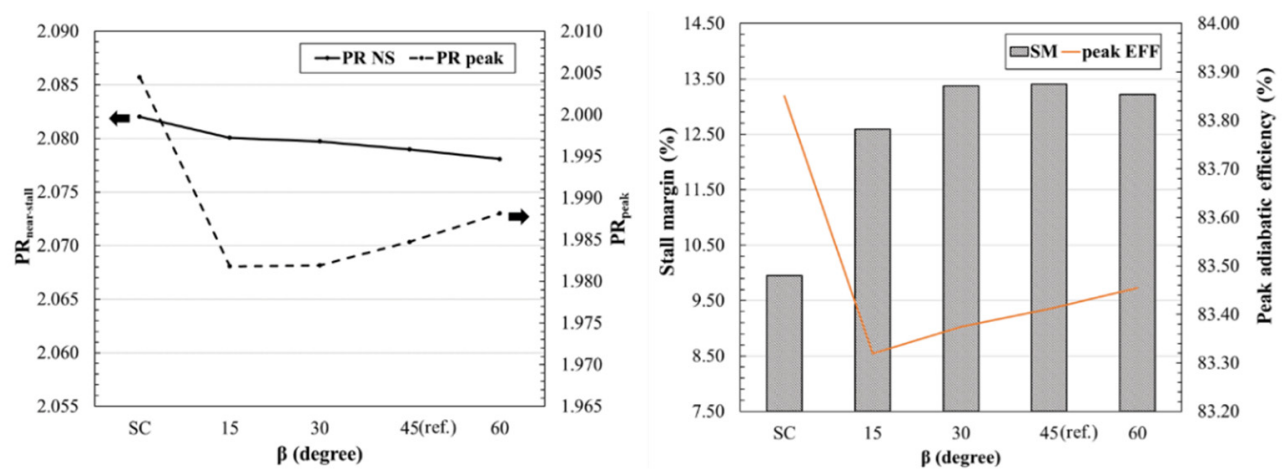

Figure 15. Effects of rear bleed angle on performance.

Figure 16 compares the levels of influence of all tested geometric parameters on the compressor's stall margin. This comparison shows that three parameters $\left(\mathrm{L}_{\mathrm{F}}, \mathrm{L}_{\mathrm{B}}, \alpha\right)$ have the highest sensitivity on the stabilizing effect of the RE2B channel. Therefore, they were selected as design variables for the subsequent optimization, while the other geometric parameters were fixed. 


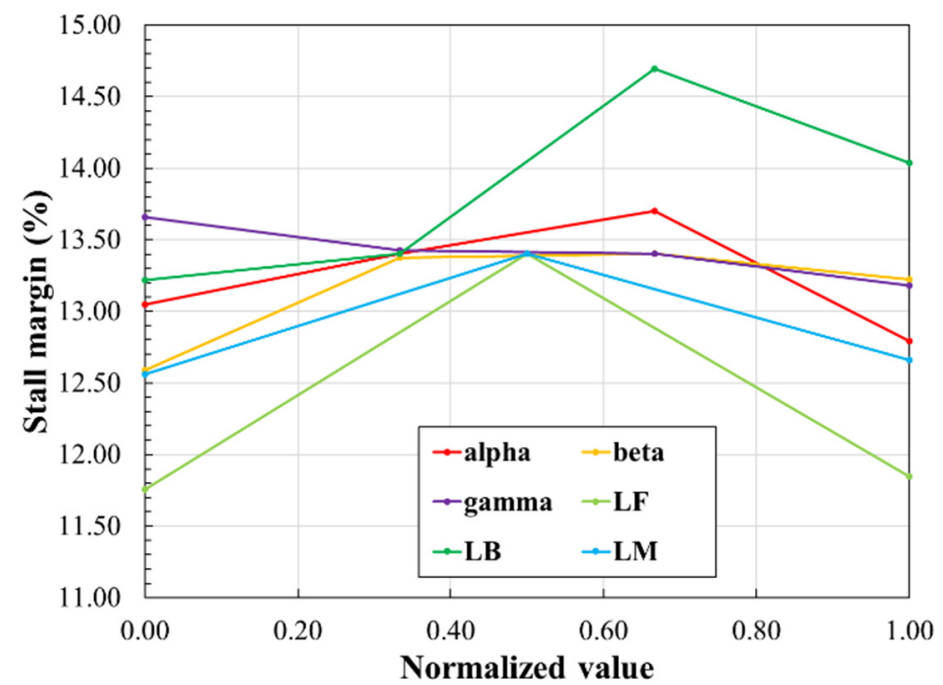

Figure 16. Sensitivity analysis of geometric parameters.

\subsection{Optimization}

A single-objective optimization has been performed with three design variables, $\mathrm{L}_{\mathrm{F}}$, $\mathrm{L}_{\mathrm{B}}$, and $\alpha$, to maximize the stall margin by RE2B. The design space, i.e., the ranges of the design variables and the values of the other parameters are specified in Table 4 . The fixed values of the parameters $\left(\mathrm{L}_{M} / \mathrm{C}_{\mathrm{R}}, \beta\right.$, and $\gamma$ ) are their best values for stall margin improvement found from the parametric study. The DOE has 20 design points generated using LHS. RANS simulations were performed to obtain the stall margin values at these design points. Based on those data, an RBNN model was created to approximately relate the stall margin to the design variables. The spread constant and the sum squared error goal for the RBNN model were 0.1 and 0.00002 , respectively.

Table 4. Design space for optimization.

\begin{tabular}{ccccccc}
\hline & \multicolumn{3}{c}{ Design Variables } & \multicolumn{3}{c}{ Fixed Parameters } \\
\cline { 2 - 7 } & $\mathbf{L}_{\mathbf{F}} / \mathbf{C}_{\mathbf{R}}(\mathbf{\%})$ & $\mathbf{L}_{\mathbf{B}} / \mathbf{C}_{\mathbf{R}}(\mathbf{\%})$ & $\boldsymbol{\alpha}\left({ }^{\circ}\right)$ & $\mathbf{L}_{\mathbf{M}} / \mathbf{C}_{\mathbf{R}}(\mathbf{\%})$ & $\beta\left({ }^{\circ}\right)$ & $\gamma\left({ }^{\circ}\right)$ \\
\hline Lower limit & 40 & 70 & 45 & 55 & 45 & 15 \\
Upper limit & 50 & 80 & 75 & 55 & & \multirow{2}{*}{} \\
\hline
\end{tabular}

Using the PSO algorithm, the optimal design (PSO.opti) was found at $\mathrm{L}_{\mathrm{F}} / \mathrm{C}_{\mathrm{R}}=46.25 \%$, $\mathrm{L}_{\mathrm{B}} / \mathrm{C}_{\mathrm{R}}=77.57 \%$, and $\alpha=46.56^{\circ}$. The new design provided a stall margin of $15.04 \%$, which is a significant increase of $51.16 \%$ from that of the smooth casing case. In Tables 5 and 6 , to assess the effectiveness of optimization, the optimal design is compared with a new reference design (nREF). For the nREF design, $\mathrm{L}_{\mathrm{F}}, \mathrm{L}_{\mathrm{B}}$, and $\alpha$ are set at the reference values in Table 1 , while $L_{M}, \beta$, and $\gamma$ are fixed at the values shown in Table 4. The optimum design improves the stall margins of SC and nREF by $51.16 \%$ and $9.22 \%$, respectively. This remarkable achievement validates the benefit of performing optimization. In terms of accuracy, the difference in the stall margin between the prediction by MATLAB and RANS simulation is only $0.07 \%$. This small error indicates that the combination of the RBNN model and PSO algorithm is accurate in finding the optimal design for this problem. 
Table 5. Optimization results.

\begin{tabular}{|c|c|c|c|c|c|c|c|c|}
\hline \multirow{2}{*}{ Design } & \multirow{2}{*}{$\mathrm{L}_{\mathrm{F}} / \mathrm{C}_{\mathrm{R}}(\%)$} & \multirow{2}{*}{$\mathrm{L}_{\mathrm{B}} / \mathrm{C}_{\mathrm{R}}(\%)$} & \multirow{2}{*}{$\alpha\left({ }^{\circ}\right)$} & \multicolumn{2}{|c|}{ Objective Function } & \multirow{2}{*}{ Error $(\%)$} & \multicolumn{2}{|c|}{ Improvement (\%) } \\
\hline & & & & MATLAB & RANS & & vs. SC & vs. nREF \\
\hline SC & - & - & - & - & 9.95 & - & - & - \\
\hline nREF & 45 & 70 & 45 & - & 13.77 & - & 38.39 & - \\
\hline PSO.opti & 46.25 & 77.57 & 46.56 & 15.05 & 15.04 & -0.07 & 51.16 & 9.22 \\
\hline
\end{tabular}

Table 6. Comparison of peak efficiency and pressure ratio between optimal design and other cases.

\begin{tabular}{ccccccc}
\hline \multirow{2}{*}{ Design } & \multirow{2}{*}{$\boldsymbol{\eta}_{\text {peak }}(\mathbf{\%})$} & PR $_{\text {peak }}$ & $\mathbf{P R}_{\text {NS }}$ & \multicolumn{3}{c}{ Changes vs. SC (\%) } \\
\cline { 5 - 7 } & & & & $\eta_{\text {peak }}$ & PR $_{\text {peak }}$ & PR $_{\text {NS }}$ \\
\hline SC & 83.85 & 2.0045 & 2.0820 & - & - & - \\
nREF & 83.41 & 1.9778 & 2.0785 & -0.52 & -1.33 & -0.17 \\
PSO.opti & 83.39 & 1.9562 & 2.0762 & -0.55 & -2.41 & -0.28 \\
\hline
\end{tabular}

Figure 17 compares PSO.opti and nREF in terms of the rotor's tip loading at the peak efficiency condition. At this condition, PSO.opti shows that a shockwave is located further downstream, and the pressure difference at the upstream region is smaller compared to that of nREF. As a result, the flow becomes more stable and the compressor's stable working range extends to a higher normalized mass flow rate of 0.9953 (the values for SC and nREF are 0.9934 and 0.9939 , respectively).

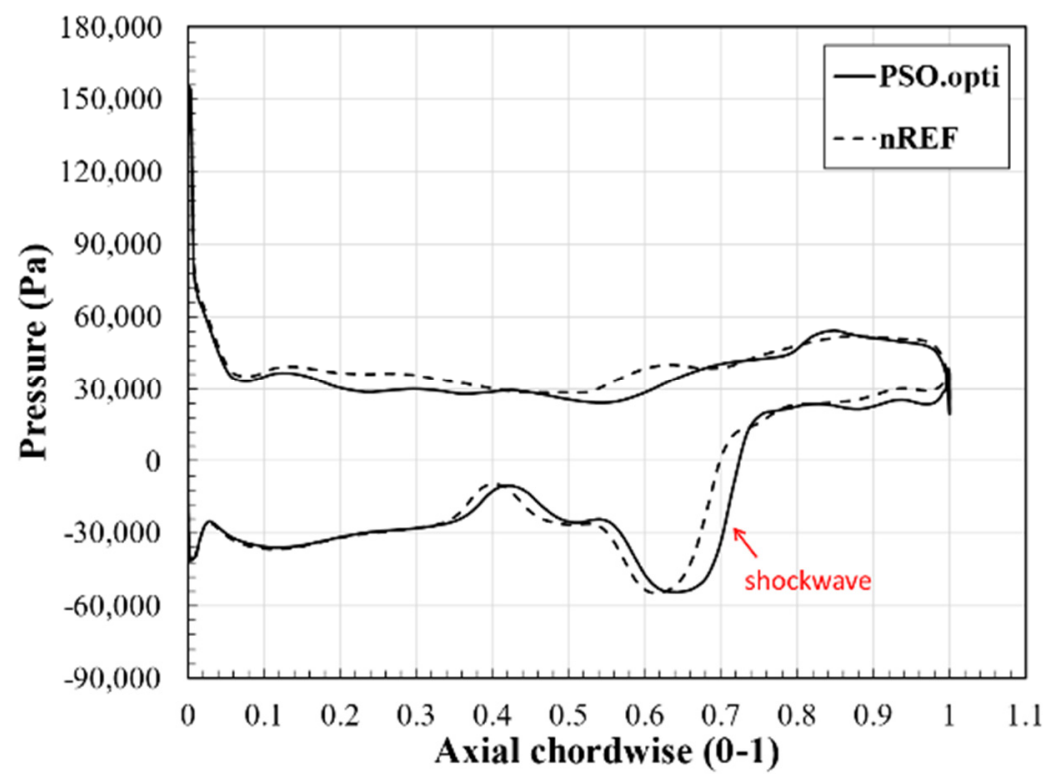

Figure 17. Blade loading at $99 \%$ rotor span at peak adiabatic efficiency condition.

Figure 18 shows the Mach number distribution at the near-stall condition. While there is no significant difference between the two cases in the rotor domain, the stagnation zones in the stator domain are smaller than those in the case of PSO.opti, which is related to the improvement in flow stability. The combination of this change and the improvement at the peak efficiency condition mentioned above seems to be the main reason for the higher stall margin with the PSO.oti design. 


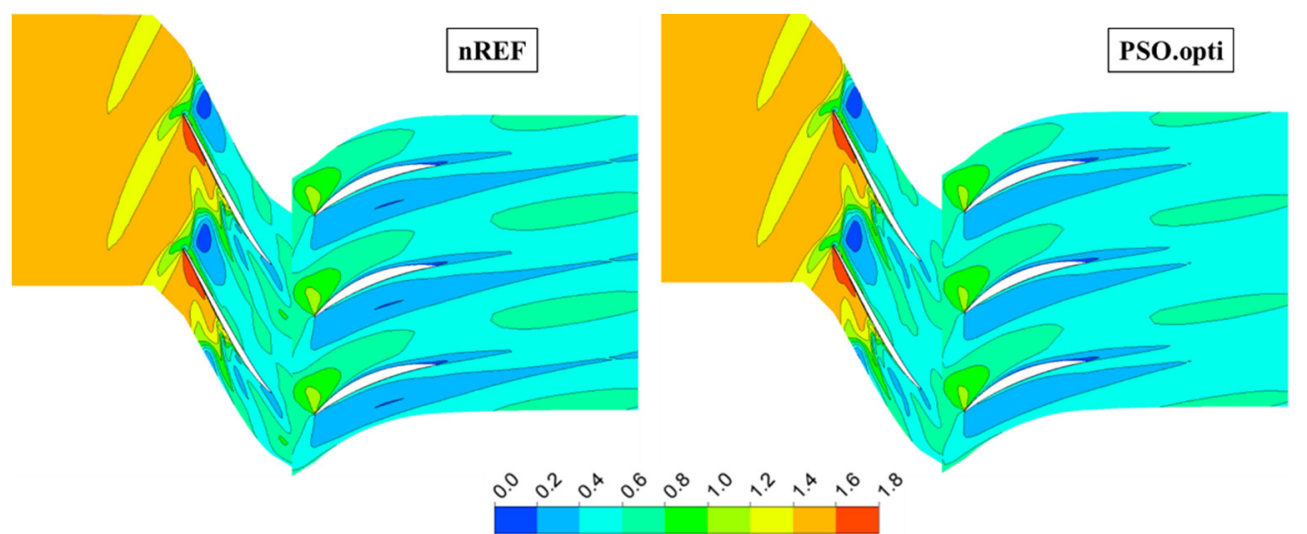

Figure 18. Mach number contours at $98 \%$ blade span at near-stall conditions.

\section{Conclusions}

The effects of the RE2B channel on the aerodynamic performance and stability of the single-stage transonic axial compressor, NASA Stage 37 were investigated using 3D RANS analysis. Numerical results confirmed the RE2B's ability to improve the stability of the transonic compressor, with only small reductions in the efficiency and pressure ratio. The reference RE2B design increased the compressor's stall margin from $9.95 \%$ of the smooth casing to $13.40 \%$ with a less than $1 \%$ reduction in the peak efficiency and pressure rise. As the conventional single-channel design (RE), RE2B showed the stabilizing effects on the compressor's flow field: Delaying stagnation zones' expansion and distancing flow separation from the leakage vortex. However, with the additional bleeding channel, the RE2B channel removed a sizable stagnation zone near the rotor's trailing edge shown in the case of RE. This improvement might be the main reason the stall margin with RE2B is approximately $8.33 \%$ higher than that with RE.

From the parametric study, the injection location, injection angle, and rear bleeding location are found to be the most influential parameters on the compressor's performance among the tested six geometric parameters of RE2B. By selecting those three parameters as design variables, single-objective optimization using the PSO algorithm combined with the RBNN surrogate model was conducted to maximize the stall margin improvement. With only $0.55 \%$ and $0.28 \%$ reductions, respectively, in the peak adiabatic efficiency and maximum pressure ratio, the optimal design at $\mathrm{L}_{\mathrm{F}} / \mathrm{C}_{\mathrm{R}}=46.25 \%, \mathrm{~L}_{\mathrm{B}} / \mathrm{C}_{\mathrm{R}}=77.57 \%$, and $\alpha=46.56^{\circ}$, provided a large stall margin of $15.04 \%$, i.e., $51.16 \%$ and $9.22 \%$ increases from those of the smooth casing and the reference design, respectively.

In future work, the study of RE2B needs to be expanded to its effects on aeromechanic and aero-acoustic characteristics to provide a more comprehensive assessment of its applicability to real compressors.

Author Contributions: T.-D.V. presented the main idea of the dual-bleeding recirculation casing treatment for the axial transonic compressor, analyzed the proposed casing treatment, and performed numerical analysis. K.-Y.K. suggested improvements for the idea and revised and finalized the manuscript. All authors have read and agreed to the published version of the manuscript.

Funding: This work was supported by the National Research Foundation of Korea (NRF) grant funded by the Korean government (MSIT) (No. 2019R1A2C1007657).

Institutional Review Board Statement: Not applicable.

Informed Consent Statement: Not applicable.

Data Availability Statement: Not available.

Conflicts of Interest: The authors declare no conflict of interest. 


\section{References}

1. Hathaway, M.D. Self-recirculating casing treatment concept for enhanced compressor performance. In American Society of Mechanical Engineers; International Gas Turbine Institute, Turbo Expo (Publication) IGTI: Amsterdam, The Netherlands, 2002; Volume 5, pp. 411-420.

2. Koff, S.; Mazzawy, R. Case treatment for compressor blades. US005282718A, 1 February 1994.

3. Hobbs, D.E.; Windsor, S. Active Vaned Passage Casing Treatment. US005431533A, 11 July 1995.

4. Nolcheff, N.A. Flow Aligned Plenum Endwall Treatment for Compressor Blades. US005586859A, 24 December 1996.

5. Strazisar, A.J.; Bright, M.M.; Thorp, S.; Culley, D.E.; Suder, K.L. Compressor stall control through endwall recirculation. In Proceedings of the ASME Turbo Expo 2004, Vienna, Austria, 14-17 June 2004; Voluem 5, pp. 655-667.

6. Khaleghi, H.; Tousi, A.M.; Boroomand, M.; Teixeira, J.A. Recirculation casing treatment by using a vaned passage for a transonic axial-flow compressor. Proc. Inst. Mech. Eng. Part A J. Power Energy 2007, 221, 1153-1162. [CrossRef]

7. Weichert, S.; Day, I.; Freeman, C. Self-regulating casing treatment for axial compressor stability enhancement. In Proceedings of the ASME Turbo Expo 2011, Vancouver, BC, Canada, 6-10 June 2011; Volume 7, pp. 225-238.

8. Khaleghi, H. Effect of discrete endwall recirculation on the stability of a high-speed compressor rotor. Aerosp. Sci. Technol. 2014, 37, 130-137. [CrossRef]

9. Wang, W.; Chu, W.; Zhang, H.; Wu, Y. The effects on stability, performance, and tip leakage flow of recirculating casing treatment in a subsonic axial flow compressor. In Proceedings of the ASME Turbo Expo, Seoul, Korea, 13-17 June 2016; Volume 2 A-2016.

10. Dinh, C.T.; Ma, S.B.; Kim, K.Y. Effects of a circumferential feed-back channel on aerodynamic performance of a single-stage transonic axial compressor. Proc. ASME Turbo Expo 2017, 2A-2017, 57-65. [CrossRef]

11. Li, J.; Du, J.; Li, Z.; Lin, F. Stability enhancement with self- recirculating injection in axial flow compressor. J. Turbomach. 2018, 140, 071001. [CrossRef]

12. Khaleghi, H. A new approach of endwall recirculation in axial compressors. Aerosp. Sci. Technol. 2020, 98, 105704. [CrossRef]

13. Vuong, T.; Kim, K.; Dinh, C. Recirculation-groove coupled casing treatment for a transonic axial compressor. Aerosp. Sci. Technol. 2021, 111, 106556. [CrossRef]

14. Samad, A.; Kim, K.Y. Shape optimization of an axial compressor blade by multi-objective genetic algorithm. Proc. Inst. Mech. Eng. Part A J. Power Energy 2008, 222, 599-611. [CrossRef]

15. Deb, K.; Pratap, A.; Agarwal, S.; Meyarivan, T. A Fast and Elitist Multiobjective Genetic Algorithm: NSGA-II. IEEE Trans. Evol. Comput. 2002, 6, 182-197. [CrossRef]

16. Lucas, J.M. Response Surface Methodology: Process and Product Optimization Using Designed Experiments, 3rd edition. J. Qual. Technol. 2010, 42, 228-230. [CrossRef]

17. Deb, K. Multi-objective Optimisation Using Evolutionary Algorithms: An Introduction. In Multi-Objective Evolutionary Optimisation for Product Design and Manufacturing; Wang, L., Ng, A.H.C., Deb, K., Eds.; Springer: London, UK, 2011; pp. 3-34. ISBN 978-0-85729-652-8.

18. Kim, J.-H.; Ovgor, B.; Cha, K.H.; Kim, J.H.; Lee, S.; Kim, K.Y. Optimization of the aerodynamic and aeroacoustic performance of an axial-flow fan. AIAA J. 2014, 52, 2032-2044. [CrossRef]

19. Khalfallah, S.; Ghenaiet, A.; Benini, E.; Bedon, G. Surrogate-Based Shape Optimization of Stall Margin and Efficiency of a Centrifugal Compressor. J. Propuls. Power 2015, 31, 1607-1620. [CrossRef]

20. Ma, S.B.; Afzal, A.; Kim, K.Y. Optimization of ring cavity in a centrifugal compressor based on comparative analysis of optimization algorithms. Appl. Therm. Eng. 2018, 138, 633-647. [CrossRef]

21. Goldberg, D.E. Genetic algorithms in search, optimization, and machine learning. Choice Rev. Online 1989, 27, 27-0936. [CrossRef]

22. Kennedy, J.; Eberhart, R. Particle swarm optimization. In Proceedings of the ICNN'95-International Conference on Neural Networks, Perth, Australia, 27 November-1 December 1995; Volume 4, pp. 1942-1948.

23. Van Laarhoven, P.J.M.; Aarts, E.H.L. Simulated Annealing BT-Simulated Annealing: Theory and Applications; van Laarhoven, P.J.M., Aarts, E.H.L., Eds.; Springer: Dordrecht, The Netherlands, 1987; pp. 7-15. ISBN 978-94-015-7744-1.

24. Cheng, J.; Zhao, S.; Dong, Z.; Yang, C. Design Optimization of a Multistage Axial Flow Compressor Based on Full-Blade Surface Parameterization and Phased Strategy. Int. J. Aerosp. Eng. 2021, 2021. [CrossRef]

25. Jinxin, C.; Chen, J.; Xiang, H. Aerodynamic Optimization Design of Compressor Blades Based on Improved Artificial Bee Colony Algorithm. In Proceedings of the 2018 Joint Propulsion Conference, Cincinnati, OH, USA, 9-11 July 2018; AIAA Propulsion and Energy Forum. American Institute of Aeronautics and Astronautics: Reston, VA, USA, 2018.

26. Yan, S.; Chu, W.L. Influence of self-circulating casing treatment with double-bleed ports structure on compressor performance. Proc. Inst. Mech. Eng. Part G J. Aerosp. Eng. 2020, 234, 1743-1756. [CrossRef]

27. Lonnie, R.; Moore, R.D. Design and Overall Performance of Four Highly Loaded, High-Speed Inlet Stages for an Advanced High-Pressure-Ratio Core Compressor. In NASA Technical Paper 1337; NASA Lewis Research Center: Cleverland, OH, USA, 1978.

28. Wilke, I.; Kau, H.P.; Brignole, G. Numerically aided design of a high-efficient casing treatment for a transonic compressor. In Proceedings of the ASME Turbo Expo, Reno-Tahoe, NV, USA, 6-9 June 2005; Volume 6, pp. 353-364.

29. Hofmann, W.; Ballmann, J. Tip clearance vortex development and shock-vortex-interaction in a transonic axial compressor rotor. In Proceedings of the 40th AIAA Aerosp. Sci. Meet. Exhib, Reno, NV, USA, 14-17 January 2002. [CrossRef]

30. ANSYS. ANSYS CFX-Solver Theory Guide Release 15.0; ANSYS: Canonsburg, PA, USA, 2014 
31. Shim, H.-S.; Kim, S.-H.; Kim, K.-Y. Analysis and Optimization of Staggered Partial Diffuser Vanes in a Centrifugal Pump. J. Fluids Eng. 2020, 142, 051207. [CrossRef]

32. Barth, T.; Jespersen, D. The design and application of upwind schemes on unstructured meshes. In Proceedings of the 27th Aerospace Sciences Meeting; Aerospace Sciences Meetings, Reno, NV, USA, 9-12 January 1989; American Institute of Aeronautics and Astronautics: Reston, VA, USA, 1989.

33. Chen, H.; Huang, X.; Fu, S. CFD investigation on stall mechanisms and casing treatment of a transonic compressor. In Proceedings of the Collection of Technical Papers-AIAA/ASME/SAE/ASEE 42nd Joint Propulsion Conference, Sacramento, CA, USA, 9-12 July 2006; Volume 6, pp. 4789-4798.

34. Kim, J.H.; Choi, K.J.; Kim, K.Y. Performance evaluation of a transonic axial compressor with circumferential casing grooves. In Proceedings of the Institution of Mechanical Engineers. Part A J. Power Energy 2012, 226, 218-230. [CrossRef]

35. Dinh, C.T.; Heo, M.W.; Kim, K.Y. Aerodynamic performance of transonic axial compressor with a casing groove combined with blade tip injection and ejection. Aerosp. Sci. Technol. 2015, 46, 176-187. [CrossRef]

36. Celik, I.; Karatekin, O. Numerical experiments on application of richardson extrapolation with nonuniform grids. J. Fluids Eng. Trans. ASME 1997, 119, 584-590. [CrossRef]

37. McKay, M.D.; Beckman, R.J.; Conover, W.J. A Comparison of Three Methods for Selecting Values of Input Variables in the Analysis of Output from a Computer Code. Technometrics 1979, 21, 239-245. [CrossRef]

38. MATLAB. The Language of Technical Computing-Release 14; Mathworks: Natick, MA, USA, 2004. 